\title{
Caring for the Patient with an Epidural in the Recovery Unit
}

\author{
Sharon E. Kitcatt \\ Consultant Nurse - Acute Pain Service Ashford and St Peter's Hospitals NHS Trust
}

$\mathrm{T}$ his article is a summary of a presentation given at the BARNA conference in Hove in June 2006 and is drawn from the practical experience of the writer in helping recovery staff to manage epidural analgesia.

Epidural analgesia can provide excellent post-operative pain relief. Nursing staff in Recovery Units caring for patients with this method of post-operative analgesia must receive appropriate training and support to help them to care safely for these patients. Patients also need to feel cared for and safe in the hands of staff that they have confidence in and a core role of an Acute Pain Service is to educate and support staff to enable them to do this.

Drugs injected into the epidural space will result in both central and systemic absorption. Epidurals for postoperative pain are usually administered as a continuous infusion (controlled epidural analgesia, CEA) or a combination of continuous infusion with patient administered boluses (patient controlled epidural analgesia, PCEA). Most hospitals use a combination of opioids and local anaesthetic agents and at Ashford and St Peter's Hospitals NHS Trust, our epidural solution also contains adrenaline. Adrenaline can enhance the action of opioid and local anaesthetic agents at a spinal level, improving pain relief and sensory block (Niemi and Breivik, 1998). The constrictive effect of adrenaline can also reduce the systemic effects of fentanyl such as sedation (Niemi and Breivik, 1998).

An effective epidural should provide the patient with relief from dynamic pain, i.e. pain during coughing, moving, deep breathing, thereby helping to reduce post-operative complications. Poorly managed acute pain

Correspondence to: Sharon E Kitcatt, Consultant Nurse - Acute Pain Service, Ashford and St Peter's Hospitals NHS Trust, Surrey KT16 OPZ, UK. E-mail: Sharon.Kitcatt@asph.nhs.uk; Tel: 01932722281. could also be harmful to the patient due to the stimulation of the sympathetic nervous system. Epidurals containing a local anaesthetic can block the effects of the sympathetic nervous system which may provide other benefits in addition to analgesia such as improved pulmonary function and a reduction in thrombotic complications. However, there are also risks involved with this invasive procedure and the patient's anaesthetist will consider these and discuss them with the patient before inserting an epidural.

The Recovery Unit is the best place to sort out problems with an epidural. If an epidural is working safely and effectively in the Recovery Unit, then the chances of the epidural continuing to work for the patient is increased. In addition to being able to manage pain, Recovery Unit nurses must know how to trouble shoot epidural problems and how to set up and manage epidural equipment.

When an epidural is not working in the Recovery Unit, we have often heard an anaesthetist say 'but it was working in theatre....' This can be for several reasons including the administration of the initial loading dose and also the patient's position during surgery. However, this does not guarantee that the epidural with continue to work. An epidural that is not working in the Recovery Unit must be managed or the patient given an alternative method of analgesia. The administration of opioids by another route when a patient is still receiving epidural analgesia is not a safe option as this could lead to delayed, unpredictable respiratory depression. In addition, the patient may gain relief from the pain from the additional analgesia which still leaves an epidural that is not functioning which will be a problem again once the patient is back on the ward. The exception to this is for patients who usually take analgesics such as tramadol, codeine or morphine; such patients may have a higher opioid requirement post-operatively and should maintain 
their usual opioid intake in addition to the epidural. If an epidural is not working, check the epidural site for leakage and check the sensory level before further troubleshooting is undertaken.

The sensory test is looking for a change in sensation, using ice or pinprick, not simply whether the patient can just feel touch or pressure. Some weaker epidural solutions may not provide a definite block but most patients can indicate a change. The sensory level should be just above the level of the incision. If the patient is in pain and the sensory level is too low, then first consider increasing the epidural infusion rate, and changing the patient's position. The sensory block should be no higher than nipple level as this may compromise cardiac or respiratory function. If higher than this, you need to consider why this is. Catheter migration is possible or have they just had a top up? Or were they in a head down position in theatre as this could cause the block to rise due to gravity. If the sensory block is above nipple level (thoracic level 4[T4]) then check baseline observations (checking particularly for hypotension), check for any tingling/ weakness in arms/hands, and any respiratory difficulties. If any of these are present, it may indicate that the catheter has migrated spinally or the epidural has responded to gravity due to the position in theatre. Reassure the patient, stop the epidural and sit patient up if possible. Check all connections in case the epidural has been attached to an IV line, inform the anaesthetist, get fluids reviewed (see below) and keep patient in the Recovery Unit until the block is at block down to T4.

Hypotension can occur without any of the above accompanying signs. Hypotension can be due to sympathetic blockade but the most common cause is fluid depletion and the nurse should follow their usual checks to ascertain whether fluid is needed, including the monitoring of the patient's urine output. Smith (2003) suggests that a low urine volume, i.e. $30 \mathrm{~mL} / \mathrm{hr}$ (or $0.5 \mathrm{~mL} / \mathrm{kg} / \mathrm{hr}$ ) should not go untreated or uninvestigated for more than two consecutive hours.

An epidural sited at the lumbar level may cause a degree of motor block. Patients who have a thoracic epidural should not have a motor block and the presence of this may indicate a problem with the epidural. If the patient has had a loading dose of high concentration of a local anaesthetic or they have had a combined spinal epidural, then this could produce a temporary motor block. If the patient is awake, then they should be asked to move their legs and feet and if this test is not possible due to the patient being sleepy, then this should be communicated to the ward staff collecting the patient.

\section{DEVELOPING EPIDURAL SKILLS IN THE RECOVERY UNIT}

Nursing in a Recovery Unit provides you with a great opportunity to learn how to manage epidural analgesia. Recovery Unit nurses should be confident to care for patients who have had epidural 'top ups', including the necessary observations and the possible side effects including the drugs that may be needed to manage these.

To care for the patient with an epidural safely, the Recovery Unit nurse must understand how epidurals work including the related pharmacology. Ideally, the nurse should attend an annual epidural educational update in addition to attending Acute Pain Service ward rounds and using reflection to enable them to challenge practice. Practical skills that cannot be gained easily in the Recovery Unit setting can be assessed using scenarios, role play, etc. Nurses should also be familiar with any Trust Guidelines for epidural management including equipment, drugs such as anticoagulants in relation to epidurals. It is also important to have an awareness of national guidance so that epidural services can be benchmarked against these. Examples are guidelines from the Royal College of Anaesthetists, Royal College of Nursing (2004) and NHS Quality Improvement Scotland (2004).

To summarise, get the patient comfortable before they leave the Recovery Unit, document their pain score remembering to use dynamic pain assessment which is pain on moving, coughing or deep breathing. Make sure that all epidural information is communicated to and checked with ward staff including the prescription, other documentation and the pump settings. Ensure that a physical check is made with the ward staff relating to the patient's physical condition relating to the epidural such as motor function and the epidural line and site. In addition, ensure that acute pain principles are followed: check that the patient has regular, simple analgesia and anti-emetics prescribed, and that the epidural is prescribed properly.

Ward epidurals are now routine in many trusts. At Ashford and St Peter's Hospitals NHS Trust, we have dedicated equipment for epidurals, adrenaline in our epidural solution as standard, and we have now started using PCEA in our surgical high dependency area. It is too early to make decisions about rolling this out to the wards, but our early experience has shown that we rarely have to administer epidural 'top ups' and patients generally seem to like having control over their epidural. 


\section{REFERENCES}

NHS Quality Improvement Scotland. Best Practice Statement - Postoperative Pain Management. NHS Quality Improvement Scotland, 2004. www.nhshealthquality.org

Niemi G, Breivik H. Adrenaline markedly improves thoracic epidural analgesia produced by a low-dose infusion of bupivacaine, fentanyl and adrenaline after major surgery. Acta Anaesthesiologica Scandinavica 1998; 42: 897-909.

Royal College of Anaesthetists, Royal College of Nursing. Good Practice in the Management of Continuous Epidural Analgesia in the Hospital
Setting. Royal College of Anaesthetists, London. 2004. www.rcoa.ac.uk

Smith G. ALERT - A Multiprofessional Course in the Care of the Acutely Ill Patient. Portsmouth Institute of Medicine, Faculty of Health and Social Care, 2003. 